\title{
Quantal Problems with Partial Algebraization of the Spectrum
}

\author{
M. A. Shifman and A. V. Turbiner \\ Institute of Theoretical and Experimental Physics, Moscow SU-117259, USSR
}

\begin{abstract}
We discuss a new class of spectral problems discovered recently which occupies an intermediate position between the exactly-solvable problems (e.g., harmonic oscillator) and all others. The problems belonging to this class are distinguished by the fact that a part of the eigenvalues and eigenfunctions can be found algebraically, but not the whole spectrum. The reason explaining the existence of the quasi-exactly-solvable problems is a hidden dynamical symmetry present in the hamiltonian. For one-dimensional motion this hidden symmetry is $S L(2, R)$. It is shown that other groups lead to a partial algebraization in multidimensional quantal problems. In particular, $S L(2, R) \times S L(2, R)$, $S O(3)$ and $S L(3, R)$ are relevant to two-dimensional motion inducing a class of quasi-exactly-solvable two-dimensional hamiltonians. Typically they correspond to systems in a curved space, but sometimes the curvature turns out to be zero. Graded algebras open the possibility of constructing quasi-exactlysolvable hamiltonians acting on multicomponent wave functions. For example, with a (non-minimal) superextension of $S L(2, R)$ we get a hamiltonian describing the motion of a spinor particle.
\end{abstract}

\section{Introduction}

Recent investigations [1-7] of the spectral problem of Schrödinger type have led to a surprising finding: a new type of problem has been discovered in which a part of the spectrum can be found by purely algebraic methods (quasi-exactly-solvable problems according to terminology of [4]). In the present paper we develop the approach proposed in $[2,3,6]$. The main idea lying in the basis of this approach is the existence of a hidden dynamical symmetry inherent to the hamiltonians of quasiexactly-solvable type.

Any hamiltonian $H$ can obviously be represented as an infinite-dimensional hermitian matrix,

$$
H \Rightarrow\left[\begin{array}{ccccc}
h_{11} & h_{12} & \ldots & h_{1 n} & \ldots \\
h_{21} & h_{22} & \ldots & h_{2 n} & \ldots \\
\ldots \ldots \ldots \ldots & \ldots & \ldots & \ldots \\
h_{n 1} & h_{n 2} & \ldots & h_{n n} & \ldots \\
\ldots & \ldots & \ldots & \ldots & \ldots
\end{array}\right] .
$$


To this end choose an arbitrary complete set of orthonormal functions $\psi_{i}$ satisfying the boundary conditions; then define

$$
h_{i j}=\left\langle\psi_{i}|H| \psi_{j}\right\rangle \text {. }
$$

Generally speaking, all $h_{i j}$ 's will be non-vanishing.

In this language the solution of the spectral problem reduces to a diagonalization of the infinite-dimensional matrix $\left\{h_{i j}\right\}$. The eigenfunctions of the hamiltonian are the eigenvectors of the matrix $\left\{h_{i j}\right\}$. Unfortunately, unlike the case of the finite matrices, there are no general algebraic rules which would allow one to diagonalize the infinite-dimensional matrix $\left\{h_{i j}\right\}$. (The exactly solvable problems are distinguished by the fact that in these problems the matrix $\left\{h_{i j}\right\}$ is very specific and is reducible to the diagonal form with the aid of an algebraic procedure. The most well-known example is, of course, the harmonic oscillator.)

Assume, however, that the matrix $\left\{h_{i j}\right\}$ has a block structure:

$$
H \Rightarrow\left\{h_{i j}\right\}=\left[\begin{array}{cccc|cccc}
h_{11} & h_{12} & \ldots & h_{1 N} & 0 & 0 & 0 & \ldots \\
h_{21} & h_{22} & \ldots & h_{2 N} & 0 & 0 & 0 & \ldots \\
\ldots & \ldots & \ldots & \ldots & \ldots & \ldots & \ldots & \ldots \\
h_{N 1} & h_{N 2} & \ldots & h_{N N} & 0 & 0 & 0 & \ldots \\
\hline 0 & 0 & \ldots & 0 & \multicolumn{4}{|c}{\text { Non-vanishing }} \\
0 & 0 & \ldots & 0 & \multicolumn{4}{|c}{h_{i j} \text { 's }} \\
\ldots & \ldots & \ldots & \ldots &
\end{array}\right],
$$

where $N$ is some fixed integer. The block in the upper left corner is an $N$ by $N$ matrix while the second non-vanishing block (in the lower right corner) is an infinitedimensional matrix. Then, quite obviously, one can immediately diagonalize the finite block without touching the infinite one. The operation is performed just in the same way as for any finite matrix and is purely algebraic. In other words, in the case (3) we analytically determine a part of the spectrum $-N$ eigenvalues and the corresponding eigenfunctions of the hamiltonian $H$-by algebraic manipulations.

There is a regular method which allows one to guarantee the block structure (3). Consider a finite-dimensional group $G$ possessing finite-dimensional representations $R_{j}$. Let us denote the generators of the group $G$ by $T^{a}$. If we are able to reduce the hamiltonian $H$ to a combination of the generators $T^{a}$ with constant coefficients, say,

$$
H \Rightarrow \sum_{a, b} C_{a b} T^{a} T^{b}+\sum_{a} C_{a} T^{a}
$$

$\left(C_{a b}, C_{a}\right.$ are numerical coefficients) then this hamiltonian will automatically have the desired form (3), provided that the basis in Eq. (2) is chosen as follows:

$$
\left.\left\{\left|\psi_{i}\right\rangle\right\}=\text { \{elements of } R_{j}+\text { arbitrary set from orthogonal space }\right\} .
$$

The reason is obvious: the action of the generators $T^{a}$ on the elements of $\left\{R_{j}\right\}$ leaves us inside the same representation. The dimension of the finite block in Eq. (3) coincides with that of $R_{j}$.

Thus, the central point is the possibility of reducing the hamiltonian to a combination of the generators. Normally the quantal hamiltonian is a hermitian 
differential operator of the second order. For instance, for the standard Schrödinger equation $H \psi\left(x_{i}\right)=E \psi\left(x_{i}\right)$,

$$
H=-\frac{1}{2}\left(\partial / \partial x_{i}\right)^{2}+V\left(x_{i}\right)
$$

Here the cartesian coordinates $x_{i}$ are used for definiteness ${ }^{1}, V\left(x_{i}\right)$ is the potential energy, $\psi\left(x_{i}\right)$ is the wave function. The normalizability condition is implied

$$
\int\left|\psi\left(x_{i}\right)\right|^{2} \prod_{i} d x_{i}=1
$$

On the other hand the Lie groups also admit differential realizations on the space of the inhomogeneous polynomials. The simplest group, $S L(2, R)$, can be realized on the space of polynomials of one variable. In this case one arrives at a class of hamiltonians (3) describing one-dimensional motion. Exhaustive analysis of 1-dim quasi-exactly-solvable problems has been given previously [6]. Here we generalize the approach and consider two-dimensional problems of the type (3). The following three groups are relevant in this case: $S L(2, R) \times S L(2, R), S O(3)$ and $S L(3, R)$. The corresponding generators can be written as follows ( $\xi$ and $\eta$ are two independent variables).

(i) $\operatorname{SL}(2, R) \times S L(2, R)$

This group is isomorphic to $S O(4)$, with six generators,

$$
\begin{aligned}
& T^{+}=-2 j \xi+\xi^{2} \partial_{\xi}, \quad \tilde{T}^{+}=-2 \tilde{j} \eta+\eta^{2} \partial_{\eta}, \\
& T^{0}=-j+\xi \partial_{\xi}, \quad \tilde{T}^{0}=-\tilde{j}+\eta \partial_{\eta}, \\
& T^{-}=-\partial_{\xi}, \quad \tilde{T}^{-}=-\partial_{\eta} \text {. }
\end{aligned}
$$

(ii) $S O(3)$

$$
\begin{aligned}
& T^{1}=\eta \partial_{\xi}-\xi \partial_{\eta}, \quad T^{2}=\partial_{\eta}+\eta^{2} \partial_{\eta}+\xi \eta \partial_{\xi}-j \eta \\
& T^{3}=-\partial_{\xi}-\xi^{2} \partial_{\xi}-\xi \eta \partial_{\eta}+j \xi
\end{aligned}
$$

These three generators can be obtained as linear combinations of some of the $S L(3, R)$ generators [see below, Eq. (10)]. Thus, the differential realization of $S O(3)$ presented here forms a subgroup of $\operatorname{SL}(3, R)$.

(iii) $S L(3, R)$

There are eight generators, six off diagonal ones are conveniently numbered by two indices,

$$
\begin{aligned}
& T_{3}^{1}=\eta^{2} \partial_{\eta}+\xi \eta \partial_{\xi}-j \eta, \quad T_{2}^{1}=\xi^{2} \partial_{\xi}+\xi \eta \partial_{\eta}-j \xi, \\
& T_{3}^{2}=-\eta \partial_{\xi}, \quad T_{1}^{2}=-\partial_{\xi}, \quad T_{1}^{3}=-\partial_{\eta}, \quad T_{2}^{3}=-\xi \partial_{\eta}, \\
& T_{d}=\eta \partial_{\eta}+2 \xi \partial_{\xi}-j, \quad \widetilde{T}_{d}=2 \eta \partial_{\eta}+\xi \partial_{\xi}-j .
\end{aligned}
$$

[For the case of $S L(3, R)$ Eq. (10) does not give the most general differential realization; the latter needs three, not two variables. The realization presented here

\footnotetext{
${ }^{1}$ We reserve the notation $x_{i}$ for the coordinates in the flat space; the coordinates in the curved space will be denoted by $\xi^{i}$
} 
is sufficient, however, for our present purposes - analysis of two-dimensional quasiexactly solvable systems.]

Now, the task is to find connection between the quantal hamiltonians and the generators (8)-(10).

To this end it is necessary to make one further step. The point is that the hamiltonian (6) can not be equal, by itself, to the combination of generators (4). [Notice that there is no equality sign in Eq. (4).] The equality becomes valid only after a certain transformation of $H$ and $\psi$ which we will call the "gauge" transformation keeping in mind, however, that actually it belongs to a class of nonunitary transformations and, thus, is not a genuine gauge transformation. The quotation marks are to remind us about this fact.

Consider the Schrödinger equation. Substitute the original wave function $\psi$ by a transformed wave function $\tilde{\psi}$,

$$
\psi(x)=\widetilde{\psi}(x) e^{-a(x)} .
$$

If the phase $a(x)$ were purely imaginary we would have the standard gauge transformation. Here we will deal, however, with the real phase.

Of course, Eq. (6) is non-invariant under the local transformation (11). The wave function $\tilde{\psi}$ obeys a new spectral equation,

$$
H_{(a)} \tilde{\psi}=E \tilde{\psi}, \quad H_{(a)}=-\frac{1}{2}\left(\partial / \partial x_{i}-\mathscr{A}_{i}(x)\right)^{2}+V(x), \quad \mathscr{A}_{i}=\partial a / \partial x_{i} .
$$

The hamiltonian $H_{(a)}$ can be considered as a "gauge"-transformed version of the original hamiltonian $H$, while $\mathscr{A}(x)$ is an analogue of the vector potential in electrodynamics. Instead of the Schrödinger wave function $\psi\left(x_{i}\right)$ two functions $\widetilde{\psi}\left(x_{i}\right)$ and $a\left(x_{i}\right)$ are introduced in Eq. (11), but to compensate for this the gauge freedom is present now. Equation (12), substituting the original Eq. (6), is obviously invariant under further "gauge" transformations,

$$
\tilde{\psi}\left(x_{i}\right) \rightarrow \tilde{\psi}\left(x_{i}\right) e^{-b\left(x_{i}\right)}, \quad \mathscr{A}_{i} \rightarrow \mathscr{A}_{i}+\partial b / \partial x_{i} .
$$

Any "gauge" is suitable; having solved Eq. (12) for the arbitrary "gauge" one can always return back to the Schrödinger equation which corresponds to the gauge $\mathscr{A}_{i}=0$.

The information on the probability amplitude is now coded in two functions, $\widetilde{\psi}(x)$ and $a(x)$, and can be arbitrarily redistributed between them. The "gauge" freedom allows one to realize the most convenient choice of $a(x)$. The standard possibility is $a(x)=0$. Then $\widetilde{\psi}(x)=\psi(x)$ is the Schrödinger wave function. On the contrary, one can trivialize $\tilde{\psi}(x) \equiv 1$ referring all dynamical information to the "imaginary phase" $a(x)$. The well-known Ricatti equation emerges in this way for one degree of freedom.

Following Ref. 6 we will use the "gauge" freedom and try to fix the "gauge" in such a way that the gauge-transformed hamiltonian $H_{(a)}$,

$$
H_{(a)}=\sum_{a, b} C_{a b} T^{a} T^{b}+\sum_{a} C_{a} T^{a} .
$$

This point is the last (but not the least) conceptual element of the method allowing one to construct quasi-exactly-solvable hamiltonians on a regular basis. 
Having established the relation (13) we may be sure that the original hamiltonian $H$ will have $N$ eigenvalues and eigenfunctions which can be found algebraically (recall that $N$ is the dimension of the representation $R_{j}$ ). These $N$ levels, of course, do not exhaust the whole spectrum. Apart from the algebraized sector there exists, as a rule, an infinite sequence of discrete levels of conventional (non-algebraic) nature.

Since the transformation (11) is not a real gauge transformation due to its nonunitary nature, the boundary conditions and the normalization condition for the original wave function $\psi$ and for $\tilde{\psi}$ differ. This trivial fact should always be taken into account.

We have found interesting families of quasi-exactly-solvable problems typically referring to the motion in two-dimensional curved space. Unfortunately, the analysis we have carried out is not exhaustive, and we are planning to continue it further, and also consider the motion in three and more dimensions. Notice that some multidimensional problems in curved spaces are discussed in works [8] within another approach.

The organization of the paper is as follows. In Sect. 2 we give a general pattern for consideration of the multidimensional quantal problems. Section 3 demonstrates how the groups $S U(2) \times S U(2), S O(3)$ and $S L(3, R)$ can be used for constructing two-dimensional hamiltonians with the algebraized part of the spectrum. Section 4 is devoted to graded algebras and the multicomponent Schrödinger-type equations. In Sect. 5 we give a brief summary of results and list unsolved questions.

\section{Generalities}

Partial algebraization of the spectrum observed in $[1,4,5]$ in one-dimensional problems is not specific for $d=1$. The idea bears a general character, and if there are appropriate differential realizations of the finite groups involving more than one variable [see Eqs. (8)-(10)] it is obviously possible to construct a multidimensional differential operator of the second order, quadratic in the generators of the group. Unfortunately, unlike the one-dimensional case, this operator does not always have the form relevant to the Schrödinger-type equation (see the constraint (23) and the discussion following this relation).

Let us explain the point in more detail. From the explicit expression for the generators $T^{a}$ given in Eqs. (8)-(10) it is clear that the combination

$$
\sum_{a, b} C_{a b} T^{a} T^{b}+\sum_{a} C_{a} T^{a}
$$

does not always contain the second derivatives

$$
\partial_{i} \partial_{j} \equiv \partial^{2} / \partial \xi^{i} \partial \xi^{j}
$$

with the unit coefficient. In the general case, for arbitrary choice of $C$ 's, the derivatives $\partial_{i} \partial_{j}$ enter being multiplied by functions of $\xi^{i}$. Thus, the expression of the type $g^{i j} \partial_{i} \partial_{j}$ naturally appears where $g^{i j}$ plays the role of a metric. In other words, the straightforward generalization of the one-dimensional procedure of [6] leads us (in 
two, three, etc. dimensions) to consideration of motion in a gravitational background,

$$
-\frac{1}{2}\left[\frac{1}{\sqrt{g}} \partial_{i}\left(\sqrt{g} g^{i k} \partial_{k} \psi\right)\right]+V \psi=E \psi,
$$

where the term in the square bracket is the covariant laplacian substituting the ordinary laplacian of the flat space. As usual,

$$
g=\operatorname{det}\left\{g_{i k}\right\}=1 / \operatorname{det}\left\{g^{i k}\right\} \text {. }
$$

Moreover, after the phase transformation $\psi=\tilde{\psi} \exp (-a)$ we, evidently, arrive at

$$
\left[-\frac{1}{2} g^{i k} \partial_{i} \partial_{k}-\frac{1}{2}\left(\partial_{i} g^{i k}\right) \partial_{k}-\frac{1}{2}\left(\partial_{i} \ln \sqrt{g}\right) g^{i k} \partial_{k}+g^{i k} \mathscr{A}_{i} \partial_{k}+\Delta V\right] \tilde{\psi}=E \tilde{\psi}
$$

where

$$
\begin{gathered}
\mathscr{A}_{i}=\partial_{i} \quad a=\partial a / \partial \xi^{i}, \\
\Delta V \equiv V-\left\{\frac{1}{2} g^{i k} \mathscr{A}_{i} \mathscr{A}_{k}-\frac{1}{2}\left(\partial_{i} \ln \sqrt{g}\right) g^{i k} \mathscr{A}_{k}-\frac{1}{2} g^{i k}\left(\partial_{i} \mathscr{A}_{k}\right)-\frac{1}{2}\left(\partial_{i} g^{i k}\right) \mathscr{A}_{k}\right\} .
\end{gathered}
$$

It is convenient to introduce a new auxiliary "vector potential"

$$
\tilde{\mathscr{A}_{i}}=\partial_{i}(\ln \sqrt{g})-2 \mathscr{A}_{i}=\partial_{i}(\ln \sqrt{g}-2 a) \text {. }
$$

Then the spectral Eq. (16) takes the form

$$
-2 H_{(a)} \tilde{\psi} \equiv\left[g^{i k} \partial_{i} \partial_{k}+\left(\partial_{i} g^{i k}\right) \partial_{k}+\tilde{\mathscr{A}_{i}} g^{i k} \partial_{k}-2 \Delta V\right] \tilde{\psi}=-2 E \tilde{\psi}
$$

Now, let us compare the left-hand side of Eq. (20) with the combination (13) where the generators $T^{a}$ are taken in the differential form [see e.g. Eqs. (8)-(10)]. Assume that the coefficients $C_{a b}$ are chosen in a certain way. First of all, $C_{a b} T^{a} T^{b}$ contains the term $\partial_{i} \partial_{k}$ with the coefficient depending on the set of $C_{a b}$ 's. We identify the latter coefficient with the metric $g^{i k}$. Thus, the set of the coefficients $C_{a b}$ uniquely fixes the metric $g^{i k}$. The bilinear piece $C_{a b} T^{a} T^{b}$ produces some terms linear in $\partial_{k}$ as well. Next, we add the terms $C_{a} T^{a}$ and demand the linear in $\partial_{k}$ part of $C_{a b} T^{a} T^{b}+C_{a} T^{a}$ to be equal to

$$
\left[\left(\partial_{i}+\tilde{\mathscr{A}_{i}}\right) g^{i k}\right] \partial_{k},
$$

[see Eq. (20)]. Since $g^{i k}$ is already fixed this requirement gives us $n$ equations on $\tilde{\mathscr{A}_{i}}$,

$$
\left(\partial_{i}+\tilde{\mathscr{A}_{i}}\right) g^{i k}=M^{k}, \quad k=1,2, \ldots, n,
$$

where $M^{k}$ are the coefficients in front of $\partial_{k}$ in $C_{a b} T^{a} T^{b}+C_{a} T^{a}, n$ is the number of variables $\xi^{i}$.

Finally, the terms in $C_{a b} T^{a} T^{b}+C_{a} T^{a}$ with no derivatives should be identified, according to Eq. (20), with $-2 \Delta V$.

For non-degenerate metrics ${ }^{2}$ Eq. (21) can always be solved,

$$
\tilde{\mathscr{A}_{i}}=g_{i k}\left(M^{k}-\partial_{m} g^{m k}\right), \quad i=1,2, \ldots, n \text {. }
$$

${ }^{2}$ In this paper we limit ourselves only to this case 
This solution is not the end of the story, however. Certainly, Eq. (22) ensures the desired form of $H_{(a)}$, Eq. (13), but we must remember that actually at our disposal is only one gauge freedom, one phase $a$. The functions $\tilde{\mathscr{A}_{i}}$ must be pure gauge [Eq. (19)]. This requirement imposes the following constraints on $\tilde{\mathscr{A}_{i}}$ :

$$
\partial_{k} \tilde{\mathscr{A}_{i}}-\partial_{i} \tilde{\mathscr{A}_{k}}=0
$$

to be considered as self-consistency condition. If and only if the functions $\tilde{\mathscr{A}_{i}}$ given in Eq. (22) are pure gauge can we speak of the quasi-exactly-solvable problem of the Schrödinger type.

At present no general solution of the constraints (23) are known (at least, we are aware of no such solution). For some specific choices of the coefficients $C$ the result for $\tilde{\mathscr{A}_{i}}$ turns out to be pure gauge automatically. Several such examples will be discussed below.

In this respect the situations in one dimension and in two (or three, etc.) dimensions are drastically different. The one-dimensional case could be described in the same terms as here, but in one-dimensional problems we deal with only one potential $\mathscr{A}$, and the metric $\left\{g^{i k}\right\}$ degenerates to a single function $g^{11}$. Hence:

- There is no self-consistency condition like (23), and $\mathscr{A}$ is always pure gauge (modulo the issue of normalizability of the resulting wave functions, which should be analysed separately);

- The metric $g^{11}$ is always reducible to the flat space by a change of variables.

These remarks explain why in Ref. 6 devoted to one-dimensional motion it turned out possible to get the flat space Schrödinger equation for an arbitrary set of the coefficients $C$ (again, leaving aside the issue of normalizability). Already in two dimensions, first of all, not every choice of $C$ 's is admissible from the point of view of the constraints (23). Second, even if $C$ 's are such that $\tilde{\mathscr{A}_{i}}$ given by Eq. (22) satisfy the consistency conditions (pure gauge) the metric $\left\{g^{i k}\right\}$ is not necessarily flat. Generally speaking, the corresponding curvature will be non-vanishing. (Certainly, there may exist some variants which correspond to the flat space, see below.) The best one can do in two dimensions with the general metric $\left\{g^{i k}\right\}$ is to transform it into a conformally flat one.

Passing to higher dimensions we increase the number of constraints (23) and the general analysis becomes more and more difficult.

\section{Two Dimensional Motion}

As has been already noted, three groups provide us with the differential realizations built on two variables, $\xi$ and $\eta$, the realizations suitable for the program outlined above. There are no other possibilities (higher groups require more than two variables). Let us denote that we adopt the following convention: $\xi$ and $\eta$ will be treated as the variables with the upper indices, $\xi^{1}=\xi, \xi^{2}=\eta$; then, according to the general rules the corresponding derivatives $\partial_{\xi}=\partial / \partial \xi, \partial_{\eta}=\partial / \partial \eta$ are to be interpreted as $\partial_{1}$ and $\partial_{2}$ with the lower indices.

The most obvious choice is the $S L(2, R) \times S L(2, R)$ group. Moreover, the fundamental representations of the $S O(3)$ and $S L(3, R)$ groups are three-dimensional. This means that one can construct differential realizations of these groups built on two variables. For $S L(3, R)$ not all representations are accessible in this way, but 
only those which are symmetrized products of the fundamental representation (such representations are marked by one integer number, see below). The realization covering all possible representations require in $\operatorname{SL}(3, R)$ at least three variables.

(i) $S L(2, R) \times S L(2, R)$

Six generators of this group are quoted in Eq. (8); in order to have finite dimensional representations it is necessary to require the parameters $j$ and $\tilde{j}$, figuring in Eq. (8) to be semi-integer. The dimension of the representation $R_{j \tilde{j}}$ is $N=(2 j+1)(2 \tilde{j}+1)$. (This is also the multiplicity of the "algebraized" levels.)

Now, the combination (13) should obviously contain the coupling of the tilded and untilded generators, because otherwise we get separated variables from the very beginning, a trivial situation which we would like to avoid. The desired "mixing" can take place only in the bilinear part $C_{a b} T^{a} T^{b}$.

One of the possibilities is

$$
\begin{aligned}
-2 H_{(a)}= & T^{+} T^{+}+T^{0} T^{0}+\tilde{T}^{+} \tilde{T}^{+}+\widetilde{T}^{0} \widetilde{T}^{0}+2\left\{-T^{+} \tilde{T}^{+}+T^{+} \widetilde{T}^{-}+\widetilde{T}^{+} T^{-}\right. \\
& \left.-T^{-} \widetilde{T}^{-}\right\}-(\tilde{c}-c)\left(T^{+}-\widetilde{T}^{+}\right)+(2 j+1) T^{0} \\
& +(2 \tilde{j}+1) \widetilde{T}^{0}-c T^{-}-\tilde{c} \widetilde{T}^{-},
\end{aligned}
$$

where $\tilde{c}$ and $c$ are arbitrary parameters. Comparing Eqs. (8), (24), and (20) we readily find the metric

$$
\left\{g^{i k}\right\}=\left[\begin{array}{ll}
\xi^{2}\left(1+\xi^{2}\right) & -\left(1+\xi^{2}\right)\left(1+\eta^{2}\right) \\
-\left(1+\xi^{2}\right)\left(1+\eta^{2}\right) & \eta^{2}\left(1+\eta^{2}\right)
\end{array}\right],
$$

and then, using the standard definitions, calculate the curvature

$$
R=2\left\{3 \xi^{2} \eta^{2} \frac{2 \xi \eta+1}{\left(\xi^{2}+\eta^{2}+1\right)^{2}}-\frac{\xi^{2} \eta^{2}-5 \xi \eta+2}{\left(\xi^{2}+\eta^{2}+1\right)}\right\} .
$$

At the next stage we collect in Eq. (24) all terms linear in $\partial_{k}$ and using Eq. (20) determine $\tilde{\mathscr{A}}_{k}$,

$$
\tilde{\mathscr{A}}_{1}=-\frac{\tilde{c}+2(2 j+1) \xi}{1+\xi^{2}}, \quad \tilde{\mathscr{A}}_{2}=-\frac{c+2(2 \tilde{j}+1) \eta}{1+\eta^{2}} .
$$

It is quite obvious that the consistency conditions (23) are satisfied. Using the definition (19) we find the phase

$$
\begin{aligned}
a= & (\tilde{c} / 2) \operatorname{arctn} \xi+(c / 2) \operatorname{arctn} \eta+(j+1 / 4) \ln \left(1+\xi^{2}\right) \\
& +(\tilde{j}+1 / 4) \ln \left(1+\eta^{2}\right)-(1 / 4) \ln \left(1+\xi 2+\eta^{2}\right)
\end{aligned}
$$

and easily check that the eigenfunctions belonging to the algebraized part of the spectrum are normalizable. This point is not absolutely trivial because the logarithmic behaviour of the phase $a$ at infinity implies the power-like, not exponential, fall off of the wave functions. Nevertheless,

$$
\int e^{-2 a}\left(\xi^{2 j} \eta^{2 \tilde{j}}\right)^{2} \sqrt{g} d \xi d \eta<\infty .
$$


As far as the potential $V$ is concerned, the final expression is rather cumbersome,

$$
\begin{aligned}
V= & -\frac{1}{8}\left[\frac{16 j(j+1)+\tilde{c} \xi(8 j+1-\tilde{c} \xi)}{\left(\xi^{2}+1\right)}+\frac{16 \tilde{j}(\tilde{j}+1)+c \eta(8 \tilde{j}+1-c \eta)}{\left(\eta^{2}+1\right)}\right] \\
& -\left[\frac{\xi \eta(\xi \eta-2)}{2\left(\xi^{2}+1\right)\left(\eta^{2}+1\right)}-\frac{\xi^{2} \eta^{2}\left(\xi^{2} \eta^{2}-18 \xi \eta+8\right)}{8\left(\xi^{2}+1\right)\left(\eta^{2}+1\right)\left(\xi^{2}+\eta^{2}+1\right)}\right. \\
& \left.+\frac{5 \xi^{4} \eta^{4}(2 \xi \eta-1)}{8\left(\xi^{2}+1\right)\left(\eta^{2}+1\right)\left(\xi^{2}+\eta^{2}+1\right)^{2}}\right]
\end{aligned}
$$

which entails, in turn, the following asymptotic behaviour:

$$
V \sim \xi^{2} \eta^{2}\left(5 \xi \eta-\xi^{2}-\eta^{2}\right) / 8\left(\xi^{2}+\eta^{2}\right)^{2}, \quad \xi, \eta \rightarrow \infty .
$$

It may be instructive to consider another problem associated with $S L(2, R)$ $\times S L(2, R)$ describing the motion in the domain $\xi>0, \eta>0$ (the potential is singular at $\xi=0$ and/or $\eta=0$ ).

Let

$$
\begin{aligned}
-2 H_{(a)}= & T^{0} T^{0}-\frac{1}{2}\left\{T^{0} T^{-}\right\}_{+}+\widetilde{T}^{0} \widetilde{T}^{0}-\frac{1}{2}\left\{\widetilde{T}^{0} \widetilde{T}^{-}\right\}_{+}-2 T^{0} \widetilde{T}^{0}-c T^{+} \\
& -(c+2 \tilde{j}-2 j) T^{0}-(j+1 / 2) T^{-}-c \widetilde{T}^{+} \\
& -(c+2 j-2 \tilde{j}) \widetilde{T}^{0}-(\tilde{j}+1 / 2) \widetilde{T}^{-},
\end{aligned}
$$

where $\{\ldots\}$ denotes hereafter the anticommutator and $c$ is an arbitrary parameter.

Then,

and the curvature

$$
\left\{g^{i k}\right\}=\left[\begin{array}{cc}
\xi(1+\xi) & -\xi \eta \\
-\xi \eta & \eta(1+\eta)
\end{array}\right]
$$

$$
R=-\frac{\xi^{2}+\eta^{2}-10 \xi \eta-1}{2(1+\xi+\eta)^{2}}
$$

For large $\xi$ and $\eta$ the curvature tends to a constant which depends, however, on the direction in the $(\xi, \eta)$ plane. We quote also the results for the phase $a$ and the potential $V$ without further comments:

$$
\begin{aligned}
a= & \frac{c}{2}(\xi \eta+\xi+\eta)-\frac{1}{4} \ln (\xi \eta(1+\xi+\eta)) \\
V= & c(\xi+\eta)\left[2 c(\xi+\eta+\xi \eta+1)-\frac{1}{2}\right]+\frac{20 \xi \eta-(\xi+\eta)^{2}}{2(\xi+\eta+1)^{2}} \\
& -\frac{\xi(\xi+1)^{2}+\eta(\eta+1)^{2}}{4 \xi \eta(\xi+\eta+1)^{2}}+2 c(j \xi+\tilde{j} \eta) .
\end{aligned}
$$

(ii) $S O(3)$.

The algebra of $S O(3)$ is locally isomorphic to that of $S L(2, R)$. The lowestdimension representation in $S O(3)$, however, is triplet. Correspondingly, the number of variables needed for the differential realization of $S O(3)$ is not less than two. Three generators (in the differential form) are given in Eq. (9). In the case at 
hand in order to have finite-dimensional representations the parameter $j$ in these formulae should be integer. The dimension of the representation $R_{j}$ and, hence, the number of the levels in the algebraized sector is $N=(1+j)\left(1+\frac{j}{2}\right)$. In the case at hand this representation is reducible for $j>1$.

Equations (9) imply that the generic form of the $S O(3)$ generators is

$$
T^{a}=h^{a i} \partial_{i}+p_{i}^{a} \xi^{i}
$$

where $h^{a i}$ are polynomial functions of $\xi, \eta$, and $p_{i}^{a}$ are constants,

$$
\begin{aligned}
& h^{11}=\eta, \quad h^{12}=-\xi, \quad p_{i}^{1}=0, \\
& h^{21}=\xi \eta, \quad h^{22}=1+\eta^{2}, \quad p_{1}^{2}=0, \quad p_{2}^{2}=-j, \\
& \mathrm{~h}^{31}=-1-\xi^{2}, \quad h^{32}=-\xi \eta, \quad p_{1}^{3}=j, \quad p_{2}^{3}=0 .
\end{aligned}
$$

Again, the complete analysis has not been carried out, and the class of the quasiexactly-solvable problems to be discussed below is a partial solution. The solution is valid for an arbitrary set of $C_{a b}$ provided that $C_{a}=0$. More general situations satisfying the consistency conditions (23) plus $C_{a} \neq 0$ are not found yet.

For arbitrary $C_{a b}$ 's and $C_{a}=0$,

$$
\begin{aligned}
-2 H_{(a)}= & C_{a b} T^{a} T^{b}=C_{a b} h^{a i} h^{b k} \partial_{i} \partial_{k}+C_{a b} h^{a i}\left(\partial_{i} h^{b k}\right) \partial_{k} \\
& +2 C_{a b} h^{a k} p_{m}^{b} \xi^{m} \partial_{k}+C_{a b} p_{i}^{a} p_{k}^{b} \xi^{i} \xi^{k}+C_{a b} h^{a i} p_{i}^{b},
\end{aligned}
$$

where the symmetricity of $C_{a b}$ has been taken into account. Notice that $H_{(a)}$, along with the $\partial_{i} \partial_{k}$ term contains also terms linear in $\partial_{k}$. This fact is important because otherwise we would automatically get separable variables.

From Eq. (38) it stems that

$$
g^{i k}=C_{a b} h^{a i} h^{b k} .
$$

Moreover, comparing Eqs. (38) and (20) and using the latter expression for $g^{i k}$, we arrive at the following relations for $\tilde{\mathscr{A}_{i}}$ :

$$
C_{a b}\left(\tilde{\mathscr{A}}_{i} h^{a i}\right) h^{b k}=C_{a b}\left(2 p_{i}^{a} \xi^{i}-\partial_{i} h^{a i}\right) h^{b k} .
$$

It is easy to see that there exists a solution satisfying

$$
\tilde{\mathscr{A}_{i}} h^{a i}=2 p_{i}^{a} \xi^{i}-\partial_{i} h^{a i}
$$

and, hence, valid for any set of $C_{a b}$ irrespective of the concrete values of these coefficients. Specifically,

$$
\tilde{\mathscr{A}_{i}}=-\frac{2 j+3}{2} \partial_{i} \ln \left(1+\xi^{2}+\eta^{2}\right) .
$$

The corresponding expression for the phase $a$ is logarithmic,

$$
a=\frac{j+3 / 2}{2} \ln \left(1+\xi^{2}+\eta^{2}\right)+\frac{1}{4} \ln g,
$$

which results in a power-like fall off of the eigenfunctions from the algebraized part 
of the spectrum. Therefore, it is necessary to check the normalizability of the wave functions.

Up to this point the coefficients $C_{a b}$ are unspecified. To have an idea of the metric and the potential $V$ let us consider several simple choices.

(a) $C_{11}=0, C_{a b}=0$ if $a \neq b, C_{22}=C_{33}=1$.

Then

$$
\left\{g^{i k}\right\}=\left[\begin{array}{ll}
\xi^{2} \eta^{2}+\left(1+\xi^{2}\right)^{2} & \xi \eta\left(2+\xi^{2}+\eta^{2}\right) \\
\xi \eta\left(2+\xi^{2}+\eta^{2}\right) & \xi^{2} \eta^{2}+\left(1+\eta^{2}\right)^{2}
\end{array}\right]
$$

With this metric, after some algebra one gets

$$
\sqrt{g}=\left(1+\xi^{2}+\eta^{2}\right)^{-1}, \quad R=-4\left(\xi^{2}+\eta^{2}+1\right) .
$$

Moreover, the potential $V$ turns out to be bilinear in $\xi^{i}$,

$$
V=-\frac{3}{8}\left(\xi^{2}+\eta^{2}\right) \text {. }
$$

It is interesting that the parameter $j$ is absent in the final answer for $V$ and, hence, we actually deal with the exactly-solvable problem. The same is valid in all other $S O$ (3) examples considered below. Actually the $j$-independence stems from the fact the parameter $j$ in the expression (9) for the $S O(3)$ generators can be "gauged" away. The variables can be separated in the polar coordinates, $\xi=r \cos \theta, \eta=r \sin \theta$. In these coordinates the metric takes the form $\left\{g^{i k}\right\}=\operatorname{diag}\left(\left(r^{2}+1\right)^{2}, r^{-2}\right)$.

(b) $C_{11}=C_{22}=C_{33}=1, C_{a b}=0$ if $a \neq b$.

$$
\left\{g^{i k}\right\}=\left[\begin{array}{cc}
\left(\xi^{2}+1\right)^{2}+\xi^{2} \eta^{2}+\eta^{2} & \xi \eta\left(\xi^{2}+\eta^{2}+1\right) \\
\xi \eta\left(\xi^{2}+\eta^{2}+1\right) & \left(\eta^{2}+1\right)^{2}+\xi^{2} \eta^{2}+\xi^{2}
\end{array}\right] .
$$

It is very interesting that this example corresponds to a constant curvature, $R=2$. The potential $V$ vanishes,

$$
V=0
$$

(c) $C_{22}=C_{33}=2 C_{23}=1$, other $C$ 's zero.

$\left\{g^{i k}\right\}=\left[\begin{array}{cc}\left(\xi^{2}+1-\xi \eta\right)^{2}+\xi \eta\left(1+\xi^{2}\right) & \left.\left(\xi^{2}+\eta^{2}-\xi \eta\right)(2 \xi \eta-1)+3 \xi \eta-1\right) / 2 \\ \left(\left(\xi^{2}+\eta^{2}-\xi \eta\right)(2 \xi \eta-1)+3 \xi \eta-1\right) / 2 & \left(\eta^{2}+1-\xi \eta\right)+\xi \eta\left(1+\eta^{2}\right)\end{array}\right]$.

The curvature corresponding to this metric is

$$
R=-4\left(\xi^{2}+\eta^{2}+1-\xi \eta\right)
$$

while the potential

$$
V=-\frac{3}{8}\left(\xi^{2}+\eta^{2}-\xi \eta\right)
$$

Comparing the results for $R$ and $V$ we see that there is no obvious substitution leading to separation of the variables.

(d) $2 C_{12}=1$, other $C$ 's zero,

$$
\left\{g^{i k}\right\}=\left[\begin{array}{cc}
\xi \eta^{2} & \eta\left(\eta^{2}+1-\xi^{2}\right) / 2 \\
\eta\left(\eta^{2}+1-\xi^{2}\right) / 2 & -\xi\left(\eta^{2}+1\right)
\end{array}\right]
$$


This example is interesting because the potential is singular,

$$
V=\frac{3}{8}\left(\xi / \eta^{2}\right)
$$

We arrive at the quasi-exactly-solvable spectral problem for the Laplace operator in the space with the curvature which is also singular,

(e) $2 C_{23}=1$, other $C$ 's zero,

$$
R=4 \xi / \eta^{2}
$$

$$
\left\{g^{i k}\right\}=\left[\begin{array}{cc}
-\xi \eta\left(1+\xi^{2}\right) & -\left(1+\xi^{2}+\eta^{2}+2 \xi^{2} \eta^{2}\right) / 2 \\
-\left(1+\xi^{2}+\eta^{2}+2 \xi^{2} \eta^{2}\right) / 2 & -\xi \eta\left(1+\eta^{2}\right)
\end{array}\right] .
$$

The results for the curvature and the potential are:

$$
R=4 \xi \eta, \quad V=\frac{3}{8} \xi \eta .
$$

Varying the set of the coefficients $C$ we have come across many other non-trivial and interesting situations. We hope to return to the issue of cataloguing the corresponding problems elsewhere.

(iii) $S L(3, R)$

From all three variants relevant to two-dimensional motion - $S L(2, R)$ $\times S L(2, R), \operatorname{SO}(3)$ and $S L(3, R)$ - the latter group seems to lead to the widest range of problems. The reason is obvious : it has the largest number of generators and their form is more versatile; hence, more possibilities.

Eight $S L(3, R)$ generators are presented in Eq. (10) where $j$ should be considered as an integer number. The multiplicity is the same as in the $S O(3)$ case, $N=(1+j)$ $\left(1+\frac{j}{2}\right)$

It is interesting to notice that in the case at hand one can construct quasi-exactlysolvable problems referring to the flat space. Let us dwell on this point in more detail.

Problem No. 1.

$$
\begin{aligned}
-2 H_{(a)}= & \left\{T_{1}^{2},\left(\frac{2}{3} \widetilde{T}_{d}-\frac{4}{3} T_{d}\right)\right\}_{+}+\left\{T_{1}^{3},\left(\frac{2}{3} T_{d}-\frac{4}{3} \widetilde{T}_{d}\right)\right\}_{+}-\frac{4 j}{3}\left(T_{1}^{2}+T_{1}^{3}\right) \\
& -16 \alpha\left(T_{2}^{1}+T_{3}^{1}\right)+8 \beta\left(\frac{1}{3} \widetilde{T}_{d}-\frac{2}{3} T_{d}\right)+8 \gamma\left(\frac{1}{3} T_{d}-\frac{2}{3} \widetilde{T}_{d}\right) \\
= & 4 \xi \partial_{\xi}^{2}+4 \eta \partial_{\eta}^{2}+2 \partial_{\xi}+2 \partial_{\eta}-16 \alpha\left(\xi^{2} \partial_{\xi}+\xi \eta \partial_{\eta}-j \xi+\eta^{2} \partial_{\eta}+\xi \eta \partial_{\xi}\right. \\
& -j \eta)-8 \beta\left(\xi \partial_{\xi}-j / 3\right)-8 \gamma\left(\eta \partial_{\eta}-j / 3\right) .
\end{aligned}
$$

The metric $\left\{g^{i k}\right\}=\operatorname{diag}(4 \xi, 4 \eta)$ is characterized by the zero curvature. The following obvious change of variables ensures the transition to cartesian coordinates $(x, y)$ :

$$
\xi=x^{2}, \quad \eta=y^{2} .
$$

Moreover, the solution of Eqs. (19), (21) is

$$
a=\alpha(\xi+\eta)^{2}+\beta \xi+\gamma \eta=\alpha r^{4}+\beta x^{2}+\gamma y^{2},
$$


where $r^{2}=x^{2}+y^{2}$. As far as the potential $V(x, y)$ is concerned, with the aid of Eq. (18) one can easily get that

$$
V=8 \alpha^{2} r^{6}+8 \alpha r^{2}\left(\beta x^{2}+\gamma y^{2}\right)+2\left(\beta^{2} x^{2}+\gamma^{2} y^{2}\right)-8 \alpha(j+1) r^{2} .
$$

Thus, we see that the hamiltonian (48) is a direct generalization of the problem discussed in [4]. The analogy goes even further because in the two-dimensional Schrödinger Eq. (6) with the potential (49) one can actually separate the variables by passing to the so-called generalized elliptic coordinates.

Now, let us pass to a more typical situation of curved spaces.

\section{Problem No. 2.}

$$
\begin{aligned}
-2 H_{(a)}= & T_{1}^{2} T_{1}^{2}+T_{3}^{2} T_{3}^{2}+T_{1}^{3} T_{1}^{3}+T_{2}^{3} T_{2}^{3}-\left\{T_{2}^{3}, T_{3}^{2}\right\}_{+}+\beta\left\{T_{1}^{2},\left(\frac{1}{6} \widetilde{T}_{d}-\frac{1}{3} T_{d}\right)\right\}_{+} \\
& +\beta T_{3}^{2} T_{1}^{2}+\beta\left\{T_{1}^{3},\left(\frac{1}{6} T_{d}-\frac{1}{3} \widetilde{T}_{d}\right)\right\}_{+}+\beta T_{2}^{3} T_{1}^{3}-\alpha\left(T_{d}+\widetilde{T}_{d}\right) \\
& -3 \alpha \beta\left(T_{3}^{1}+T_{2}^{1}\right)-\beta\left(\frac{1}{2}+\frac{j}{3}\right)\left(T_{1}^{2}+T_{1}^{3}\right) \\
= & \left(1+\eta^{2}+\beta(\xi+\eta)\right) \partial_{\xi}^{2}+\left(1+\xi^{2}+\beta(\xi+\eta)\right) \partial_{\eta}^{2} \\
& -2 \xi \eta \partial_{\xi} \partial_{\eta}-(1+3 \alpha) \eta \partial_{\eta}-(1+3 \alpha) \xi \partial_{\xi}+\beta \partial_{\xi}+\beta \partial_{\eta}-3 \alpha \beta \xi(\xi+\eta) \partial_{\xi} \\
& -3 \alpha \beta \eta(\xi+\eta) \partial_{\eta}+3 \alpha \beta j(\xi+\eta) .
\end{aligned}
$$

The coefficient of $\partial_{i} \partial_{k}$ in the latter expression determines the metric,

$$
\left\{g^{i k}\right\}=\left[\begin{array}{cc}
1+\eta^{2}+\beta(\xi+\eta) & -\xi \eta \\
-\xi \eta & 1+\xi^{2}+\beta(\xi+\eta)
\end{array}\right]
$$

For the general values of $\beta$ the expressions for the curvature and potential are monstrous, and we will not quote them here. As far as the phase $a$ is concerned it does not depend on $\beta$,

$$
a=\frac{1}{2} \ln \sqrt{g}+\frac{3 \alpha}{4}\left(\xi^{2}+\eta^{2}\right)
$$

If $\beta=0$ however the situation becomes trivial. The curvature has the form

$$
R=6 /\left(\xi^{2}+\eta^{2}+1\right)^{2}
$$

and tends to zero asymptotically. It is worth noting that although the corresponding space is non-compact, the Euler characteristic $p=\frac{1}{2 \pi} \int R(g)^{1 / 2} d \xi d \eta=2$. As for the potential, it is $j$-independent,

$$
V=\frac{9}{32} \alpha^{2} r^{2}-\frac{1}{8\left(r^{2}+1\right)}+\frac{5}{8\left(r^{2}+1\right)^{2}},
$$

where $r=\left(\xi^{2}+\eta^{2}\right)^{1 / 2}$. After simple manipulations the problem reduces to the standard 2-dim symmetric harmonic oscillator.

Finally, the last $S L(3, R)$-associated quasi-exactly-solvable problem to be discussed below describes the motion in the half-plane $\xi+\eta>\alpha$, where $\alpha$ is a numerical parameter. 
Problem No. 3.

$$
\begin{aligned}
-2 H_{(a)}= & \left\{T_{1}^{2},\left(\frac{1}{6} \widetilde{T}_{d}-\frac{1}{3} T_{d}\right)\right\}_{+}+T_{3}^{2} T_{1}^{2}+\left\{T_{1}^{3},\left(\frac{1}{6} T_{d}-\frac{1}{3} \widetilde{T}_{d}\right)\right\}_{+}+T_{2}^{3} T_{1}^{3} \\
& +2 \alpha T_{1}^{3} T_{1}^{2}+\left(\frac{1}{2}+\frac{j}{3}\right)\left(T_{1}^{3} T_{1}^{3}+T_{1}^{2} T_{1}^{2}\right) \\
& -\beta\left(T_{3}^{1}+T_{2}^{1}\right)-\beta \alpha\left(T_{3}^{2}+T_{2}^{3}\right) \\
= & (\xi+\eta) \partial_{\xi}^{2}+(\xi+\eta) \partial_{\eta}^{2}+2 \alpha \partial_{\xi} \partial_{\eta} \\
& -\beta\left(j \eta-\xi(\xi+\eta) \partial_{\xi}-\eta(\xi+\eta) \partial_{\eta}+j \xi\right) \\
& +\partial_{\xi}+\partial_{\eta}+\beta \alpha\left(\eta \partial_{\xi}+\xi \partial_{\eta}\right) .
\end{aligned}
$$

Reading the metric off Eq. (56) we find

$$
\left\{g^{i k}\right\}=\left[\begin{array}{cc}
\xi+\eta & \alpha \\
\alpha & \xi+\eta
\end{array}\right] .
$$

Now the standard recipe for calculating the curvature yields

$$
R=-\frac{12 \alpha^{2}}{(\xi+\eta+\alpha)(\xi+\eta-\alpha)^{2}}-2 \frac{\xi+\eta+4 \alpha}{(\xi+\eta+\alpha)(\xi+\eta-\alpha)}
$$

We quote also the results for the phase $a$ and the potential $V$ :

$$
\begin{gathered}
a=\frac{1}{2} \ln \sqrt{g}-\frac{\beta}{4}\left(\xi^{2}+\eta^{2}\right) \\
V=\frac{\beta^{2}}{8}(\xi+\eta)^{3}-\frac{\beta^{2}}{4} \xi \eta(\xi+\eta)+\frac{\beta^{2}}{4}(\xi+\eta)^{2}+\frac{\beta^{2}}{4} \alpha \xi \eta+\frac{\beta}{4}(\alpha \beta+\beta+1+2 j) \\
\times(\xi+\eta)-\frac{\xi+\eta+3 \alpha}{4(\xi+\eta+\alpha)(\xi+\eta-\alpha)}-\frac{5}{4} \frac{\alpha^{2}}{(\xi+\eta+\alpha)(\xi+\eta-\alpha)^{2}} .
\end{gathered}
$$

\section{Graded Algebras}

The simple idea which forms the basis of the approach presented here allows for a whole spectrum of generalizations. One of the promising directions is a class of problems with the multicomponent wave functions. Up to now we have considered the Schrödinger-type equations on a single scalar function $\psi$. Quite often, however, one has to deal with the wave function with more than one component. For instance, such is the situation in the coupled-channel problems, in the problems describing the motion of spin- $1 / 2$ particles in magnetic fields, etc. In this section we will demonstrate that in this case there exist quasi-exactly solvable hamiltonians as well. The discussion below gives an idea on the possibilities of the method in constructing multicomponent Schrödinger-type equations with the algebraized part of the spectrum.

In order to discuss the multicomponent equations in the present context it is necessary to invoke extensions of the ordinary Lie algebras, namely the graded algebras (in the physical literature they are called also supersymmetric algebras). Here we limit ourselves to the simplest example, $U(1) \times S L(2, R / 2)$. 
In this group in addition to the standard three $S L(2, R)$ generators $T^{a}$ and one $U(1)$ generator $J$ there are four odd elements of the algebra, $Q_{\alpha}$ and $\bar{Q}_{\alpha}(\alpha=1,2)$ transforming as $S L(2, R)$ doublets. The even elements $T^{a}, J$ are characterized by the usual commutation relations,

$$
\begin{gathered}
{\left[T^{+} T^{-}\right]=-2 T^{0}, \quad\left[T^{ \pm} T^{0}\right]=\mp T^{ \pm},} \\
{\left[J T^{a}\right]=0,}
\end{gathered}
$$

while the odd elements $Q_{\alpha}$ and $\bar{Q}_{\alpha}$ anticommute according to the group law,

$$
\begin{aligned}
& \left\{\bar{Q}_{1} Q_{2}\right\}_{+}=T^{+}, \quad\left\{\bar{Q}_{2} Q_{1}\right\}_{+}=T^{-}, \quad \frac{1}{2}\left\{\bar{Q}_{1} Q_{1}-\bar{Q}_{2} Q_{2}\right\}_{+}=T^{0}, \\
& \frac{1}{2}\left\{\bar{Q}_{1} Q_{1}+\bar{Q}_{2} Q_{2}\right\}_{+}=J,
\end{aligned}
$$

expressing the fact that the product of doublet and antidoublet yields triplet plus singlet. Finally, the commutation relations between the odd and even elements of the algebra are also quite transparent,

$$
\begin{array}{lll}
{\left[Q_{1} T^{+}\right]=Q_{2},} & {\left[Q_{2} T^{+}\right]=0, \quad\left[Q_{2} T^{-}\right]=Q_{1}, \quad\left[Q_{1} T^{-}\right]=0,} \\
{\left[\bar{Q}_{1} T^{-}\right]=\bar{Q}_{2},} & \text { etc. }
\end{array}
$$

As far as the $U(1)$ charge of $Q$ and $\bar{Q}$ is concerned, it is opposite for $Q$ and $\bar{Q}$,

$$
\left[Q_{\alpha} J\right]=-\frac{1}{2} Q_{\alpha}, \quad\left[\bar{Q}_{\alpha} J\right]=+\frac{1}{2} \bar{Q}_{\alpha},
$$

so that $T^{a}$, being the bilinear product of $Q$ and $\bar{Q}$ (Eq. (63)), is neutral, see Eq. (62).

The group structure presented in Eqs. (61)-(65) is certainly not the minimal graded algebra associated with $S L(2, R)$. From the point of view of the group theory we could easily get rid of the $U(1)$ factor and manage with only two "supercharges," not four as written down in Eq. (63). The minimal version, however, does not seem suitable for quasi-exactly-solvable problems, as will be explained shortly.

Now, can one construct a differential realization of the given graded algebra? The answer is positive, but such a realization will necessarily involve the Grassmann variable $\theta$ along with the ordinary variable $\xi$. Specifically,

$$
Q=\left[\begin{array}{l}
Q_{1} \\
Q_{2}
\end{array}\right]=\left[\begin{array}{c}
\partial_{\theta} \\
\xi \partial_{\theta}
\end{array}\right], \quad \bar{Q}=\left[\begin{array}{c}
\bar{Q}_{1} \\
\bar{Q}_{2}
\end{array}\right]=\left[\begin{array}{c}
\xi \theta \partial_{\xi}-2 j \theta \\
-\theta \partial_{\xi}
\end{array}\right],
$$

where $\partial_{\xi}=\partial / \partial \xi$ and $\partial_{\theta}=\partial / \partial \theta$. We recall that $\theta$ and $\partial_{\theta}$ anticommute, so that $\theta^{2}=\partial_{\theta}^{2}=0, \partial_{\theta}(\theta f)=f-\theta \partial_{\theta} f,\left\{\partial_{\theta} \theta\right\}=1$.

It is not difficult to check that all commutation relations (61)-(65) are indeed satisfied provided that

$$
\begin{aligned}
& T^{+}=-2 j \xi+\xi^{2} \partial_{\xi}+\xi \theta \partial_{\theta}, \quad T^{0}=-j+\xi \partial_{\xi}+\frac{1}{2} \theta \partial_{\theta}, \quad T^{-}=-\partial_{\xi}, \\
& J=-j-\frac{1}{2} \theta \partial_{\theta} .
\end{aligned}
$$

Thus, the graded algebra generalizing $S L(2, R)$ is at our disposal, and the next issue to consider is that of finite dimensional representations. Inspection of the generators (66), (67) shows that the finite-dimensional representations exist 
provided $j$ is semiinteger, in perfect analogy with the situation considered in [6],

$$
R_{j, \text { graded }}=\left\{\xi^{0}, \xi^{1}, \ldots, \xi^{2 j} ; \xi^{0} \theta, \xi^{1} \theta, \ldots, \xi^{2 j-1} \theta\right\} ;
$$

the dimension of $R_{j, \text { graded }}$ is $(4 j+1)$.

Before proceeding to construction of the quasi-exactly-solvable systems associated with the given graded algebra, a remark is in order concerning the matrix representation of the Grassmann variables. The fact that the anticommuting variables can be represented in the matrix form is well-known. We will not go into generalities here, and simply formulate the answer expecting that the reader can easily convince himself/herself of the validity of all necessary properties. The recipe is as follows: substitute $\theta$ and $\partial_{\theta}$ in the generators (66), (67) by the matrices $\sigma^{+}$and $\sigma^{-}$, respectively, $\left(\sigma^{ \pm}=\left(\sigma^{1} \pm i \sigma^{2}\right) / 2\right)$ acting on two-component spinors; moreover, in the representation (68)

In the explicit form

$$
\left.\left.\left.\theta \rightarrow\right|_{\uparrow}\right\rangle=\left[\begin{array}{l}
1 \\
0
\end{array}\right],\left.\quad \partial_{\theta} \rightarrow\right|^{\downarrow}\right\rangle=\left[\begin{array}{l}
0 \\
1
\end{array}\right] .
$$

$$
\begin{aligned}
Q & =\left[\begin{array}{c}
\sigma^{-} \\
\xi \sigma^{-}
\end{array}\right], \quad \bar{Q}=\left[\begin{array}{c}
\xi \sigma^{+} \partial_{\xi}-2 j \sigma^{+} \\
-\sigma^{+} \partial_{\xi}
\end{array}\right], \\
R_{j, \text { graded }} & \left.\left.\left.\left.=\left\{\left.\xi^{0}\right|^{\downarrow}\right\rangle, \ldots,\left.\xi^{2 j}\right|^{\downarrow}\right\rangle ;\left.\xi^{0}\right|_{\uparrow}\right\rangle, \ldots,\left.\xi^{2 j-1}\right|_{\uparrow}\right\rangle\right\} .
\end{aligned}
$$

After this remark it should be clear that the quantal hamiltonian we will finally arrive at will act on a two-component wave-function.

The next steps are conceptually the stages of the program outlined in Sect. 1 with a single but important exception. Namely, we start from the Schrödinger equation

$$
\left[-\frac{1}{2} \frac{d^{2}}{d x^{2}}+\hat{V}(x)\right] \psi(x)=E \psi(x),
$$

where $\hat{V}(x)$ now is a two-by-two hermitian matrix (the caret over $V$ is to remind us about this fact) while $\psi$ is a two-component wave function,

$$
\psi=\left[\begin{array}{l}
\psi_{1} \\
\psi_{2}
\end{array}\right] .
$$

After making the "gauge" transformation we obtain the transformed hamiltonian $H_{(a)}$ and try to find the gauge in such a way that $H_{(a)}$ would reduce to a quadratic combination of the generators (66), (67). The only peculiarity is that the class of allowed "gauge" transformations becomes much wider: instead of a purely abelian substitution $\psi=\exp (-a) \tilde{\psi}$ we can invoke non-abelian transformations

$$
\psi=U \tilde{\psi},
$$

where $U$ is, generally speaking, an arbitrary two-by-two matrix. Then

where

$$
H_{(a)}=-\frac{1}{2}\left(d_{x}+\hat{\mathscr{A}}\right)^{2}+U^{-1} \hat{V} U \equiv-\frac{1}{2} d_{x}^{2}-\hat{\mathscr{A}} d_{x}+\Delta V,
$$

$$
\hat{\mathscr{A}}=U^{-1}\left(d_{x} U\right), \quad \Delta V=U^{-1} \hat{V} U-\frac{1}{2} \hat{\mathscr{A}}^{\prime}-\frac{1}{2} \hat{\mathscr{A}}^{2},
$$

and the prime denotes differentiation over $x$. 
Now, we compare Eq. (73) with $C_{a b} T^{a} T^{b}+C_{a} T^{a}$, where the set of the generators $T^{a}$ includes now all generators written down in Eqs. (66), (67). Moreover, identifying the coefficients in front of $d_{x}$ we can always find $\hat{\mathscr{A}}$ and the corresponding matrix $U$, constructing in this way a quasi-exactly-solvable hamiltonian which acts on a two-component wave function.

Unfortunately, the situation is less favourable than that described in [6]. For the $S L(2, R)$ group, any combination $C_{a b} T^{a} T^{b}+C_{a} T^{a}$ leads to a "reasonable" hamiltonian (modulo the issue of normalizability of the wave functions, of course!). In the case at hand, however, we obtain something for $H_{(a)}$, and this "something" after the reverse gauge transformation produces the potential $\hat{V}$,

$$
\hat{V}=U(\Delta V) U^{-1}+\frac{1}{2}\left(\partial^{2} U\right) U^{-1},
$$

which typically turns out to be non-hermitian. If we insist on the hermitian hamiltonian $H$ the requirement of hermiticity of $\hat{V}$ imposes an additional condition on $\hat{\mathscr{A}}$. The general solution of this constraint is unknown. Thus, we encounter the obstacle perfectly similar to that discussed in Sect. 3.

In the absence of the complete solution it seems instructive to consider particular examples. One of them is presented below. [It is worth noticing in passing that with the minimal graded algebra $S L(2, R / 1)$ no examples with the hermitian potentials have been found thus far. That is the reason why we have turned to a nonminimal supersymmetric extension of $S L(2, R)$.]

The starting hamiltonian is

$$
\begin{aligned}
H_{(a)}= & \left\{T^{0} T^{-}\right\}_{+}+2 j T^{-}-\bar{Q}_{2} Q_{1}+\alpha T^{0}-i \beta\left(-Q_{2} T^{-}+\bar{Q}_{1}+2 j Q_{1}\right) \\
& +\frac{i}{2} \alpha \beta Q_{2}-\frac{i}{2} \beta Q_{1},
\end{aligned}
$$

where $\alpha$ and $\beta$ are numerical parameters. Using Eqs. (67) and (70) we get after simple substitutions,

$$
\begin{aligned}
H_{(a)} & =-2 \xi \partial_{\xi}^{2}-\partial_{\xi}+\alpha\left(\xi \partial_{\xi}+\frac{1}{4} \sigma_{3}\right)-i \beta\left(\xi \partial_{\xi} \sigma_{1}-2 i j \sigma_{2}\right)+\frac{i}{2} \alpha \beta \xi \sigma^{-}-\frac{i}{2} \beta \sigma^{-} \\
& =-\frac{1}{2} \frac{d^{2}}{d x^{2}}+\left(\frac{1}{2} \alpha x-\frac{i}{2} \beta x \sigma_{1}\right) \frac{d}{d x}+\frac{\alpha}{4} \sigma_{3}-2 \beta j \sigma_{2}+\frac{i}{2} \alpha \beta x^{2} \sigma^{-}-\frac{i}{2} \beta \sigma^{-}
\end{aligned}
$$

(a constant term has been omitted here).

Comparison with Eq. (73) yields

$$
\hat{\mathscr{A}}=-\frac{\alpha x}{2}+\frac{i \beta x}{2} \sigma_{1}, \quad \Delta V=\frac{\alpha}{4} \sigma_{3}-2 \beta j \sigma_{2}+\frac{i \alpha \beta}{2} x^{2} \sigma^{-}-\frac{i \beta}{2} \sigma^{-}
$$

which implies, in turn, the following expression for $U$ :

$$
U=\exp \left(-\frac{\alpha x^{2}}{4}+\frac{i \beta x^{2}}{4} \sigma_{1}\right) \text {. }
$$


Now we are finally able to present the potential $\hat{V}$, a two-by-two matrix, determining a quantal system possessing an algebraized part of the spectrum,

$$
\begin{aligned}
\hat{V}= & \frac{1}{8}\left(\alpha^{2}-\beta^{2}\right) x^{2}+\sigma_{2}\left(-2 j \beta-\frac{\beta}{4}+\frac{\alpha \beta x^{2}}{4}-\frac{\alpha}{4} \operatorname{tn} \frac{\beta x^{2}}{2}\right) \cos \frac{\beta x^{2}}{2} \\
& +\sigma_{3}\left(-2 j \beta-\frac{\beta}{4}+\frac{\alpha \beta x^{2}}{4}-\frac{\alpha}{4} \operatorname{ctn} \frac{\beta x^{2}}{2}\right) \sin \frac{\beta x^{2}}{2} .
\end{aligned}
$$

It is seen that the potential $\hat{V}$ is hermitian, as we intended to get, and stable; $(4 j+1)$ eigenvalues and eigenfunctions (obviously normalizable) can be found algebraically.

\section{Conclusions}

The discovery of a new class of spectral problems [1-7] where a part of the spectrum can be found algebraically seems to be an interesting and promising development in traditional spectral theory. The sphere of applications is by no means limited to quantum mechanics. The solution of spectral problems is a necessary step in computing all types of functional determinants, the usual business in modern field theory and string theory. A complete and systematic catalogue of quasi-exactlysolvable problems, if it existed, might be of practical use. Besides that, the program is elegant by itself and presents a challenge from the point of view of pure theory.

In the one-dimensional case an exhaustive systematization can be and has been carried out [6]. In the present work we extend the program to include multidimensional problems and those referring to the multicomponent wave functions. It is worth noting that interesting results in this direction have been reported previously [8]. The approach adopted in [8] is non-algebraic and is, rather, complementary to ours. The relation between the approach we develop in the present paper and that of [8] is not completely clear thus far.

Even in two dimensions we failed to get the most general form of the quasiexactly-solvable hamiltonian, only partial solutions have been found. And still, the task does not seem hopeless. Another interesting issue we are planning to investigate is a relation between the concrete structure of the quasi-exactly-solvable hamiltonians and the geometry of the manifolds they define through the corresponding metric $g^{i k}$ (see Sect. 3). There is also a technical problem arising in the situations where one can achieve separation of the variables. In this case we effectively get onedimensional quasi-exactly-solvable problems. Can they be reduced to the catalogue given in [6]? What group underlies algebraization of the spectrum in this case? The answers to these questions have not been obtained thus far. Finally, the last intriguing question is what happens with the quasi-exactly-solvable hamiltonians when the rank of the underlying group tends to infinity.

Acknowledgements. The authors are grateful to V. A. Fateev, S. I. Gel'fand, M. A. Olshanetsky, and A. A. Rosly for valuable comments. One of the authors (M.S.) is also grateful to A. G. Ushveridze for discussions. 


\section{References}

1. Razavy, M.: Phys. Lett. 82A, 7 (1981)

2. Zaslavsky, O.B., Ulyanov, V.V.: ZhETF 87, 1984; 1724; Teor. Mat. Fiz. 71, 260 (1987)

3. Bagrov, V.G., Vshivtsev, A.S. : Preprint No 31, Tomsk Branch of the USSR Academy of Sci., Tomsk 1986

4. Turbiner, A.V., Ushveridze, A.G.: Phys. Lett. 126A, 181 (1987)

5. Turbiner, A.V.: ZhETF 94, 33 (1988), Funct. Anal. 22, 92 (1988)

6. Turbiner, A.V.: Commun. Math. Phys. 118, 467 (1988)

7. Ushveridze, A.G.: Soviet Physics - Lebedev Inst. Rep., 1988, No. 2, pp. 37, 44

8. Ushveridze, A.G. : Lebedev Institute preprint Nos. 96, 118, 133, 134, 140, 158, 190, Moscow, 1988

Communicated by B. Simon

Received December 10, 1988; in revised form May 1, 1989 
